\title{
New, simple theory-based, accurate polarization microscope for birefringence imaging of biological cells
}

\section{In Hee Shin}

Gwangju Institute of Science and Technology Department of Information and Communications 1 Oryong-dong, Buk-gu

Gwangju 500-712, Korea

\section{Sang-Mo Shin}

Gwangju Institute of Science and Technology Institute of Medical System Engineering 1 Oryong-dong, Buk-gu Gwangju 500-712, Korea

\section{Dug Young Kim}

Gwangju Institute of Science and Technology Department of Information and Communications 1 Oryong-dong, Buk-gu Gwangju 500-712, Korea

\begin{abstract}
We propose a new, simple theory-based, accurate polarization microscope for birefringence imaging of cytoskeletal structures of biological cells. The new theory lets us calculate very easily the phase retardation and the orientation of the principal axis of a particular area of a biological living cell in media by simply measuring the intensity variation of a pixel of a CCD camera while rotating a single polarizer. Just from the measured intensity maxima and minima, the amount of phase retardation $\delta$ between the fast and the slow axis of the sample area is obtained with an accuracy of $5.010 \pm 0.798 \times 10^{-3} \mathrm{rad}$. The orientation of the principal axis is calculated from the angle of the polarizer for the intensity maximum. We have compared our microscopes with two previously reported polarization microscopes for birefringence imaging of cytoskeletal structures and demonstrated the utility of our microscope with the phase retardation and orientation images of weakly invasive MCF7 and highly invasive MDA MB 231 human breast cancer cells as an example. @ 2010 Society of Photo-Optical Instrumentation Engineers. [DOI: 10.1117/1.3327280]
\end{abstract}

Keywords: imaging systems; microscopes; polarization; backgrounds; aberrations.

Paper 09008RR received Jan. 12, 2009; revised manuscript received Dec. 1, 2009; accepted for publication Dec. 3, 2009; published online Mar. 3, 2010.

\section{Introduction}

Linearly polarized light travels at one speed in one polarized direction and at another speed in another polarized direction in a birefringent material. Birefringence, first found in calcite crystals in the 1600 s, results from the anisotropic arrangement of atoms or molecules in a material. Birefringence has been observed not only in anisotropic crystals, stressed fibers, and polymers, but also in biological cells and tissues where proteins are regularly arranged in a fibrous fashion: human crystalline lens, ${ }^{1}$ retina, ${ }^{2,3}$ collagen ${ }^{4}$ and connective tissues, ${ }^{5}$ and cerebral amyloid pathologic regions. ${ }^{6}$ Phase retardation in a birefringent material is defined as the phase difference between the two orthogonal principal axes of the material. The relation between the phase retardation and the birefringence of a material measured with a polarization microscope can be mathematically expressed as

$$
\text { Phase retardation }=\frac{2 \pi}{\lambda} \cdot \Delta n \cdot t,
$$

where $\lambda, \Delta n$, and $t$ indicate wavelength of light, birefringence, and traveling distance of light in the birefringence material, respectively.

Polarization microscopy can reveal anisotropically ordered fibrous structures in biological materials without having to stain or label them. This is a big advantage of polarization microscopy, since staining or labeling a protein might affect

Address all correspondence to: In Hee Shin, Department of Information and Communications, Gwangju Institute of Science and Technology, 1 Oryongdong, Buk-gu, Gwangju 500-712, Korea. Tel: 82-62-970-3241; Fax.: 82-62-9703244,E-mail: ih-shin@gist.ac.kr its vital function. Specifically for biological applications, two different types of polarization microscopes have been reported. ${ }^{7,8}$ One type uses two liquid crystal variable phase retarders for generating four discrete input polarization states, and the other type uses two Faraday rotators for continuous rotation of linearly polarized light with respect to a sample by controlling an external magnetic field.

The first type of polarization microscope was proposed by Oldenbourg et al. ${ }^{7}$ and has been used for cell imaging applications such as the observation of dynamic behavior of cytoskeletal fine structures in the lamellipodium of nerve growth cones. ${ }^{9,10}$ Four intensity images of a biosample are measured through an analyzer while the polarization states of the incoming light to a sample are changed into four predetermined states: left circular, right circular, crossed, and parallel polarization states. From these intensity images, four Stokes polarization parameters can be calculated on a pixel-by-pixel basis. A major advantage of this polarization microscope is its fast measurement speed. This microscope employs a commercially available high-speed liquid crystal phase retarder (LCPR). Only four discrete intensity measurements are made in this microscope, whereas many measurements at different polarization angles are required in other microscopes. Due to the white beam source and the bandpass filter employed in this microscope, however, the beam coming into the LC variable phase retarders is not monochromatic. ${ }^{7,8}$ For example, the birefringence $\Delta n$ of a commercially available, nematic, LC phase retarder for wavelengths of 610 and $630 \mathrm{~nm}$ are 0.138036 and 0.137028 , respectively. ${ }^{11}$ For $\Delta \lambda$ of $20 \mathrm{~nm}$ at

$1083-3668 / 2010 / 15(1) / 016028 / 6 / \$ 25.00$ ○ 2010 SPIE 
$\lambda=630 \mathrm{~nm}$, a spread of $3 \%$ in wavelength, each LC retarder could yield an error of 0.001 in birefringence.

The second polarization microscope, with two Faraday rotators, proposed by Kuhn et al., is called a "modulated polarization microscope." The two Faraday rotators, one positioned before a specimen and the other after the specimen, are used to modulate polarization angles. Since the high current in the solenoid of Faraday rotators generates a lot of heat, cooling systems were added to the Faraday rotators of the microscope. This makes the system expensive, and the whole assembly becomes bulky, which makes it difficult to fit this system into existing laboratory microscopes.

In this paper, we propose a new, simple theory-based, easy to build, less expensive, compact microscope by single polarization modulation, and we present some birefringence images of human breast cancer cells observed under our microscope. In terms of specifications and capabilities, our microscope is compared with the two microscopes described earlier. It is well known that only discrete data points such as five- or four-phase retardation values are good enough to obtain the complete birefringence information of a sample. ${ }^{7-11}$ Even though the measurement speed of these previous methods are very fast, the accuracy in a measured phase retardation image is limited in these methods. The major advantage of our method is that more accurate phase retardation can be obtained compared to previous methods by having 30 or even more phase scanning data points and fitting them with a sine function. In general, a method for obtaining the phase retardation of a sine function by fitting a set of equally spaced phase data with a sine function is much accurate than previous methods. Since we fit a set of data with a sine function of a linearly scanned phase, there is no error related to the accuracy of a phase retarder used in conventional phase retardation measurement methods, where the phase retardation of a sine function is obtained from four or five measured intensities of a sine function at given discrete phase values.

\section{Experimental Setup}

The optical components in our polarization microscope are arranged as shown schematically in an exploded view in Fig. 1. The light source for this microscope is a randomly polarized He-Ne laser beam with 7-mW average power (1137, JDS Uniphase Corp.). In order to eliminate the problems associated with a coherent imaging system, a rotating diffuser (Korea Electro-Optics Co.) is placed just after the laser. Unwanted speckle patterns appearing in a coherent image are effectively eliminated by rotating the diffuser during measurements. ${ }^{12}$ Scattered light from the rotating diffuser is collimated with a condenser lens. This partially collimated laser beam is folded by $90 \mathrm{deg}$ with an Au-coated mirror. The elliptical envelope due to different reflectivity of the polarization components of the gold-coated mirror can be ignored. The intensity changes randomly with changing rotation angle of the polarizer, which shows that the elliptical envelope of the Au-coated mirror is negligible. A motorized rotating polarizer is placed after the mirror. Another condenser lens, placed after the rotating polarizer (linear, $\lambda=632.8 \mathrm{~nm}$; Thorlab), receives the linearly polarized beam from the polarizer and focuses it to illuminate the sample. A $60 \times$ microscope objective lens (NA: 0.85; Edmund Optics) used for imaging is

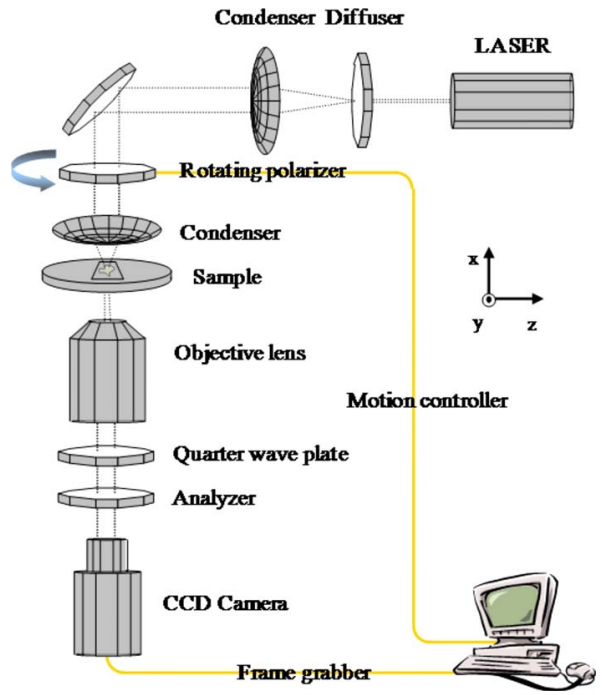

Fig. 1 Arrangement of optical components of the polarization microscope. The polarizer is rotated by a stepping motor.

placed after the sample. A quarter-wave plate (QWP, zero order, $\lambda=632.8 \mathrm{~nm}$; Thorlab) and a fixed analyzer (linear, $\lambda$ $=632.8 \mathrm{~nm}$, Thorlab) are placed just after the objective lens. The angle between the fast axis of the QWP and the polarizer is set to make $45 \mathrm{deg}$. The magnified images of a sample are detected by a CCD camera ( 8 bit, $640 \times 480$ pixels; COHU) and are digitized by a frame grabber with 8 -bit resolution. In order to remove the interference effect between the CCD sensor and its protecting cover glass, the cover just in front of the $\mathrm{CCD}$ camera is removed. ${ }^{13} \mathrm{~A}$ series of intensity images are acquired at 30 different angles of the rotating polarizer scanning from 0 to $180 \mathrm{deg}$. The intensity variation of each pixel in the series of measured images is fitted with a sine function of the input polarization angle to find the principal axis and the phase retardation of each pixel.

\section{Theory}

Figure 2 shows the relative polarization angles of the optical components in our newly proposed polarization microscope with a single rotating polarizer. The polarizer, initially set to 0 deg with respect to the $z$ axis of the diagram, rotates from $0 \mathrm{deg}$ to $180 \mathrm{deg}$. Image acquisition is synchronized to the rotation of the polarizer such that successive image frames are captured as the polarizer rotates. Let us consider a birefringent sample whose phase retardation is $\delta$ and whose fast axis is located at $\phi$ with respect to the $z$ axis. Light passes through the fixed quarter-wave plate (QWP) and the fixed analyzer before it is detected by the CCD. The polarizing angles of the fast axis of the QWP and the analyzer are set to $45 \mathrm{deg}$ and $90 \mathrm{deg}$ with respect to the $z$ axis, respectively. Jones matrix representations of the polarization state of the light just after each polarization component can be written as follows:

- Polarization state of the light after the polarizer whose angle is $\theta$ with respect to the $z$ axis:

$$
\left[\begin{array}{c}
E_{z} \\
E_{y}
\end{array}\right]=\left[\begin{array}{c}
\cos \theta \\
\sin \theta
\end{array}\right] E_{0} e^{i \varpi t}
$$




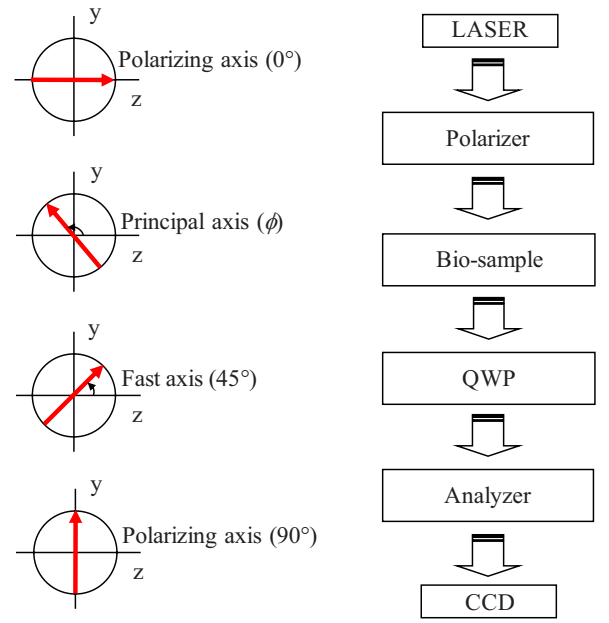

Fig. 2 Orientation angle of each polarization component of the polarization microscope. The polarizer rotates from 0 to $180 \mathrm{deg}$, while the QWP is set at 45 deg from the $z$ axis and the analyzer at 90 deg.

- Polarization state of the light just after the birefringent sample whose phase retardation is $\delta$, and its fast axis is rotated $\phi$ with respect to the $z$ axis:

$$
\left[\begin{array}{c}
E_{z}^{\prime} \\
E_{y}^{\prime}
\end{array}\right]=\left[\begin{array}{cc}
\cos \phi & -\sin \phi \\
\sin \phi & \cos \phi
\end{array}\right]\left[\begin{array}{cc}
1 & 0 \\
0 & e^{-i \delta}
\end{array}\right]\left[\begin{array}{cc}
\cos \phi & \sin \phi \\
-\sin \phi & \cos \phi
\end{array}\right]\left[\begin{array}{l}
E_{z} \\
E_{y}
\end{array}\right] .
$$

- Polarization state of the light just after the QWP whose fast axis is rotated $45 \mathrm{deg}$ with respect to the $z$ axis:

$$
\begin{aligned}
{\left[\begin{array}{l}
E_{z}^{\prime \prime} \\
E_{y}^{\prime \prime}
\end{array}\right]=} & {\left[\begin{array}{cc}
\cos \left(\frac{\pi}{4}\right) & -\sin \left(\frac{\pi}{4}\right) \\
\sin \left(\frac{\pi}{4}\right) & \cos \left(\frac{\pi}{4}\right)
\end{array}\right]\left[\begin{array}{cc}
1 & 0 \\
0 & -i
\end{array}\right] } \\
& \times\left[\begin{array}{cc}
\cos \left(\frac{\pi}{4}\right) & \sin \left(\frac{\pi}{4}\right) \\
-\sin \left(\frac{\pi}{4}\right) & \cos \left(\frac{\pi}{4}\right)
\end{array}\right]\left[\begin{array}{c}
E_{z}^{\prime} \\
E_{y}^{\prime}
\end{array}\right] .
\end{aligned}
$$

- Polarization state just after the analyzer whose fast axis is rotated $90 \mathrm{deg}$ with respect to the $z$ axis:

$$
\left[\begin{array}{l}
E_{z}^{\prime \prime \prime} \\
E_{y}^{\prime \prime \prime}
\end{array}\right]=\left[\begin{array}{ll}
0 & 0 \\
0 & 1
\end{array}\right]\left[\begin{array}{l}
E_{z}^{\prime \prime} \\
E_{y}^{\prime \prime}
\end{array}\right] \text {. }
$$

Then, the intensity of the light detected by the CCD camera as a function of the rotation angle $\theta$ of the polarizer $I(\theta)$ can be written as

$$
I(\theta) \equiv\left|E_{z}^{\prime \prime \prime}\right|^{2}+\left|E_{y}^{\prime \prime \prime}\right|^{2}=\frac{I_{0}}{2}[1+\sin \delta \cdot \sin (2 \theta-2 \phi)],
$$

where $I_{0} / 2=E_{0} E_{0}^{*} / 2$ is the average intensity of the sinusoidal intensity variation, and $\left|E_{z}^{\prime \prime \prime}\right|^{2}$ in Eq. (6) is identically zero. As seen in Eq. (6), the intensity will vary sinusoidally with respect to the angle $\theta$ of the rotating polarizer. Figure 3 illus-

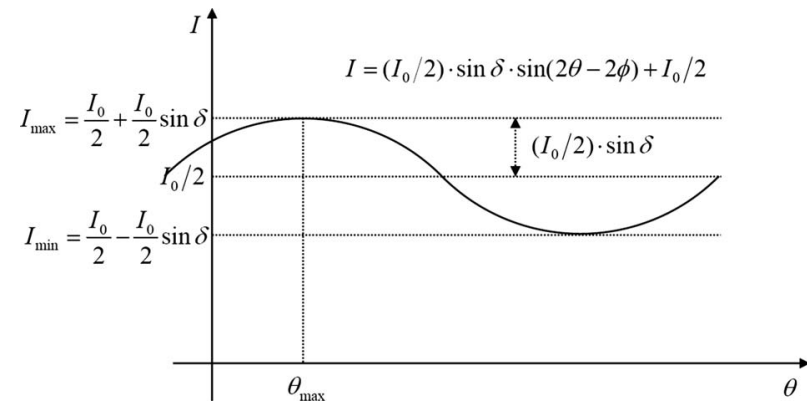

Fig. 3 Theoretical intensity of the light from a given point on a sample varies sinusoidally as the polarizer rotates from 0 to $180 \mathrm{deg}$.

trates typical sinusoidal intensity data as a function of the rotating polarizer angle. The amplitude of the sinusoidal intensity variation depends on the phase retardation $\delta$, and the average intensity $I_{0} / 2$. From Eq. (6), we can obtain the phase retardation $\delta$ as

$$
\delta=\arcsin \left(\frac{I_{\max }-I_{\min }}{I_{\max }+I_{\min }}\right) .
$$

Equation (6) indicates that the maximum intensity of measured data is obtained when $2 \theta_{\max }-2 \phi=\pi / 2$. Therefore, we can obtain the orientation angle of the principal axis of the sample as

$$
\phi=\theta_{\max }-\pi / 4,
$$

where $\theta_{\max }$ is the angle of the rotating polarizer corresponding to the maximum intensity with respect to the $z$ axis.

\section{Accuracy and Precision of the Microscope}

When the phase retardation $\delta$ is 0 in Eq. (6), the intensity should become constant, $I_{0} / 2$, even if the angle $\theta$ of the polarizer changes. The phase retardation $\delta$ becomes zero if the sample has no retardation or when there is no sample in the polarization microscope. But in reality, there is a minute,

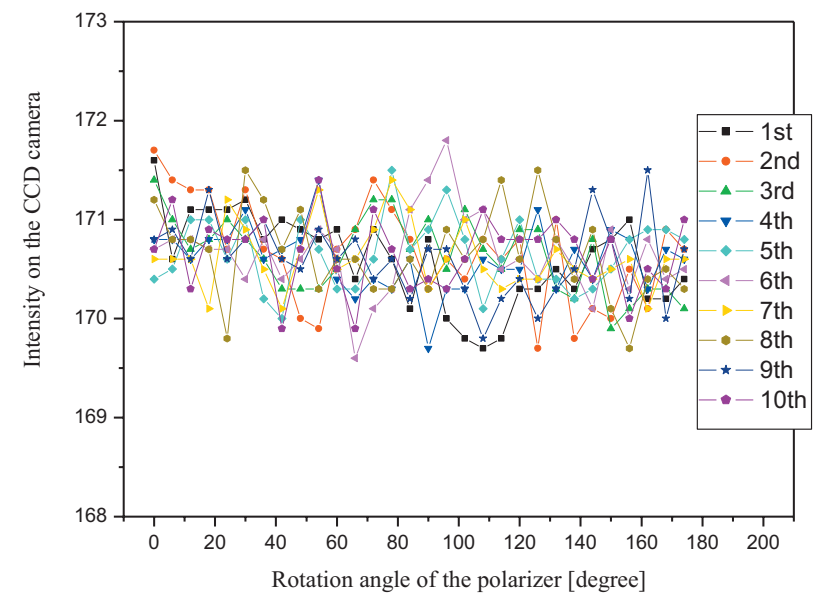

Fig. 4 Intensity variations were measured 10 times without a sample to check the accuracy and precision of the polarization microscope. Without a sample, the intensity should not change theoretically. 
Table 1 Specifications and capabilities of three types of polarization microscopes.

\begin{tabular}{|c|c|c|c|}
\hline & $\begin{array}{l}\text { Oldenbourg et al. } \\
\text { (Refs. 7, 9, and 10) }\end{array}$ & Kuhn et al. (Ref. 8) & This work \\
\hline Main components & $\begin{array}{l}1 \text { polarizer } \\
1 \text { analyzer } \\
1 \text { quarter-wave plate } \\
2 \text { liquid crystal (LC) } \\
\text { phase retarders }\end{array}$ & $\begin{array}{l}1 \text { polarizer } \\
1 \text { analyzer } \\
2 \text { Faraday rotators }\end{array}$ & $\begin{array}{l}1 \text { polarizer } \\
1 \text { analyzer } \\
1 \text { quarter-wave plate } \\
1 \text { stepping motor }\end{array}$ \\
\hline $\begin{array}{l}\text { Polarization } \\
\text { modulation }\end{array}$ & $\begin{array}{l}\text { Phase retardation } \\
\text { modulation } \\
\text { of (LCs) } \\
\text { by voltage control }\end{array}$ & $\begin{array}{l}\text { Polarization state } \\
\text { modulation of polarizer/analyzer } \\
\text { by Faraday rotators }\end{array}$ & $\begin{array}{l}\text { Polarization state } \\
\text { modulation of polarizer by rotating } \\
\text { the polarizer }\end{array}$ \\
\hline Optical source & Arc lamp light plus band pass filter & Arc lamp light plus band pass filter & He-Ne laser plus diffuser \\
\hline Source wavelength & $\begin{array}{l}\lambda c=546 \mathrm{~nm} \\
(\Delta \lambda=10 \mathrm{~nm})\end{array}$ & $\begin{array}{l}\lambda c=436 \mathrm{~nm} \\
(\text { No remark on } \Delta \lambda)\end{array}$ & $\begin{array}{l}\lambda c=632.8 \mathrm{~nm} \\
\left(\Delta \lambda \sim 10^{-3} \mathrm{~nm}\right)\end{array}$ \\
\hline Accuracy & No remark & No remark & $0.005010(\mathrm{rad})$ \\
\hline Precision & No remark & No remark & $0.000798(\mathrm{rad})$ \\
\hline $\begin{array}{l}\text { Measurement } \\
\text { speed }\end{array}$ & $\begin{array}{l}\text { Fast (milliseconds) } \\
\text { Set of four images } \\
\text { Electric control }\end{array}$ & $\begin{array}{l}\text { Fast } \\
\text { Continuous rotation } \\
\text { Electric control }\end{array}$ & $\begin{array}{l}\text { Relatively slow } \\
\text { Continuous rotation } \\
\text { Mechanical control }\end{array}$ \\
\hline $\begin{array}{l}\text { Sources of } \\
\text { potential errors }\end{array}$ & $\begin{array}{l}\Delta \lambda / \lambda \text { effect on two LC } \\
\text { retarders }\end{array}$ & $\begin{array}{l}\text { Heating effect on } \\
\text { Faraday rotators }\end{array}$ & $\begin{array}{l}\text { Intensity variation due } \\
\text { to the light scattering } \\
\text { particles in the diffuser }\end{array}$ \\
\hline
\end{tabular}

inherent fluctuation of the diffused laser beam because the particles of the diffuser scattering the coherent laser beam are not homogeneous in size and position. In addition to the intensity fluctuation of the beam source, digitizing the intensity for curve fitting can also be a source of minute error. These factors could affect the accuracy of the microscope.

We measured the intensity variation as a function of the rotating polarizer without any sample for 10 times, and the result is shown in Fig. 4. Ten images were averaged to produce one data point in each intensity plot. As expected from the random nature of the intensity variation caused by the diffusing particles, the intensity changes randomly with changing the rotation angle of the polarizer. Intensity fluctuation data shown in Fig. 4 represent all possible noise sources in our measurement system, which include laser intensity fluctuation, the effect of rotating diffuser, and digitizing error in our CCD camera with a frame grabber, etc. Even though each plot does not seem to show any periodic variation with respect to the angle of the rotating polarizer, the calculated $\delta$ by fitting it with a sine function yields nonzero phase retardation. The mean value of 10 different plots shown in Fig. 4 is $0.0050 \mathrm{rad}$, while their standard deviation is $0.00079 \mathrm{rad}$. Without a sample, $\delta$ is supposed to be zero. Since the average phase retardation is about six times larger than the standard deviation, we believe that there exists a certain amount of intrinsic birefringence in the optical measurement system used in our experiment. We believe that this nonzero birefringence of air measured by our proposed method is due to the nonuniform reflectivity of the 90-deg folding mirror used in our experimental setup. Therefore, the accuracy of $\delta$ measured in our microscope is $0.00501 \mathrm{rad}$, the minimum detectable value of phase retardation using our microscope, and its precision is 0.000798 . These values of accuracy and precision can be converted to the birefringence values of 0.000025 and 0.000004 by assuming that the thickness of the cell is $20 \mu \mathrm{m}$. Our slow measurement speed can restrict the spatial resolution of our birefringence measurement if the sample is moving. Since the measurement speed of the polarization microscope is as low as 0.05 frames/second, it takes about $20 \mathrm{~s}$ to obtain a single birefringence image. Therefore, the movement of our sample cells within the measurement time of $20 \mathrm{~s}$ needs to be less than the spatial resolution of our system, which is about $300 \mathrm{~nm}$.

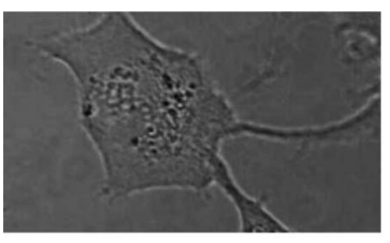

(a)

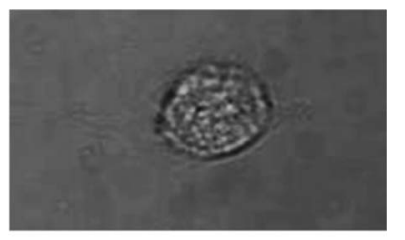

(b)
Fig. 5 Cytoskeletal structures of (a) MCF 7 and (b) MDA MB 231 breast cancer cells are not visible at all in the micrographs obtained with a conventional optical microscope. 


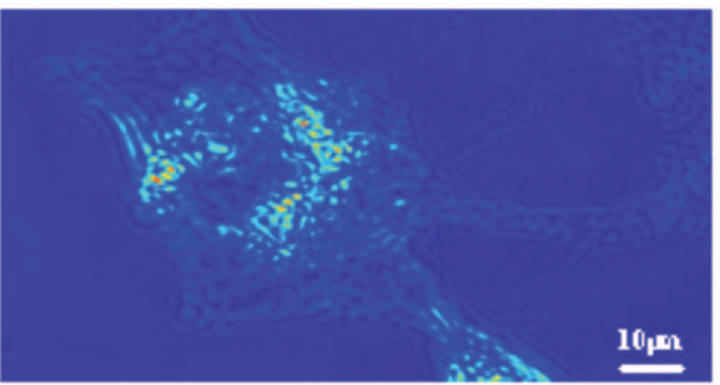

(a)

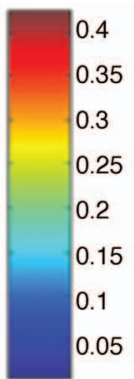

05

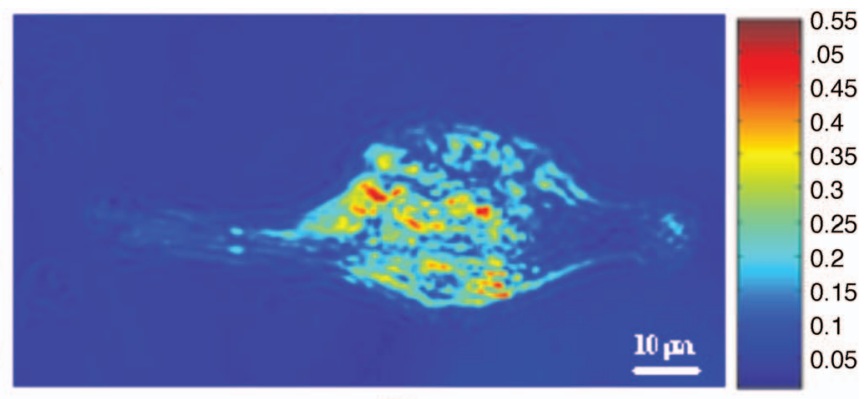

(b)

Fig. 6 Cytoskeletal structures of (a) MCF 7 and (b) MDA MB 231 breast cancer cells are clearly visible in the micrographs obtained with the polarization microscope.

\section{Comparison with Other Polarization Microscopes}

Table 1 shows a comparison of our microscopes with two other microscopes reported by Oldenbourg et al. ${ }^{7,9,10}$ and Kuhn et al., ${ }^{8}$ respectively. As briefly explained in Sec. 1 the major difference of these polarization microscopes is the modulation method of polarization. Oldenbourg et al. use two voltage-controlled LC phase retarders to make a set of four polarization states of the incoming light, and Kuhn et al. use two current controlled high-voltage Faraday rotators to continuously rotate the polarization state of the incoming light. In these microscopes, there are no mechanically moving or rotating components. Thus, the measurement speed is supposed to be fast. In our microscope, we use a stepping motor to mechanically rotate the polarizer instead of electrically or magnetically changing the polarization state of the light. Since we are not using LC retarders or Faraday rotators, our microscope is comparatively low cost, simple, and easy to build in laboratories.

Since the light sources in other microscopes are not monochromatic, this could act as a potential source of error. For the microscope by Kuhn et al., another source of error comes from the heating of Faraday rotators due to the large current flowing in the solenoids of the Faraday rotators. Although it is not mentioned in their published papers, if there were any hysteresis in the LC retarders or Faraday rotators, this could be another source of error when repeated measurements are required. Unfortunately, the accuracies of the microscopes could not be compared because the accuracies of the two microscopes were not mentioned in the papers.

\section{Birefringence Images of Human Breast Cancer Cells}

To demonstrate the utility of our microscope, human breast cancer cells from two cell lines, weakly invasive MCF 7 and highly invasive MDA MB 231, were observed for their birefringence images. The cells were selected as the samples for birefringence imaging because the cells' movement speeds are below $10 \mathrm{~nm} / \mathrm{s}$, which is appropriate for the polarization microscope with the frame rate of 0.05 frames/s. The cells were cultured for two days in the standard condition of $37{ }^{\circ} \mathrm{C}, 5 \%$ $\mathrm{CO}_{2}, 95 \%$ humidity, and in a culture medium of (Dulbecco's modified Eagle's medium DMEM; Gibco) supplemented with $10 \%$ fetal bovine serum (Hyclone) and antibodies. ${ }^{14,15}$

The micrographs in Fig. 5 are the optical images of MCF 7 [Fig. 5(a)] and MDA MB 231 [Fig. 5(b)] cells captured with a conventional microscope. The micrographs in Fig. 6 and Fig. 7 show the phase retardation distributions $\delta$ and the principal axis orientation distributions $\phi$ for these two breast cancer cells. The scale bars in Fig. 6 indicate phase retardation values in rad, and the scale bars in Fig. 7 indicate the orientation of the principal axis in radian with respect to the vertical $y$ axis.

Either microtubules ${ }^{8}$ or actin $^{9}$ filaments are known to constitute the cytoskeletal structures of cells - the sources of birefringence in cells. Even though the size of an actin filament

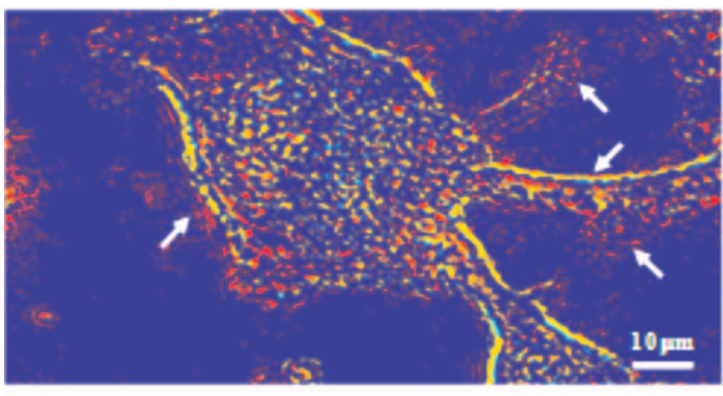

(a)

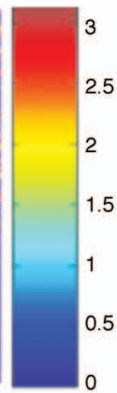

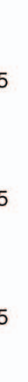
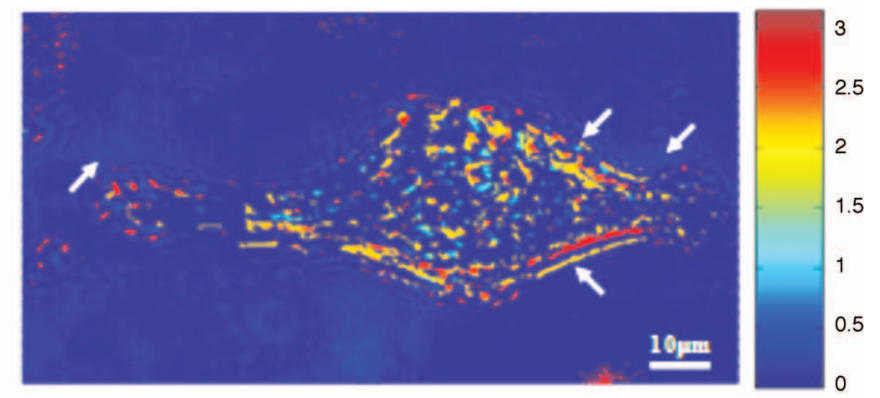

(b)

Fig. 7 These micrographs show the orientations of the principal axis of each pixel in the phase retardation micrographs shown in Fig. 6. The arrows indicate the additional features that are not seen in the phase retardation map in Fig. 6. 
or a microtubule is much smaller than the resolution of an optical microscope, our results shown in Fig. 6 and Fig. 7 indicate that the aggregated distribution of actin filaments or microtubules can produce large amount of birefringence, which can be measured by our system. Whereas the optical micrographs in Fig. 5 do not show any hints of cytoskeletal structures, the phase retardation images in Fig. 6 clearly show distribution of the cytoskeletal structures, especially in the periphery of the cells' nuclei and membranes. It is noteworthy that the principal axis plots in Fig. 7 show distribution of more structures of cells, indicated by the arrows, which are not clearly seen in the phase retardation plots in Fig. 6 .

\section{Conclusions}

In this paper, we proposed a new theory for polarization microscope imaging, compared the specifications and capabilities of our polarization microscope with two other microscopes reported previously, and demonstrated the utility of the microscope with the birefringence images of human breast cancer cell lines MCF 7 and MDA MB 231. The theory is simple in that a series of intensity measurements of a pixel on a CCD, measured while rotating a polarizer from 0 to $180 \mathrm{deg}$ at an interval of $6 \mathrm{deg}$, is fitted to a sine curve to find the maxima and minima of the curve. From the intensity maxima and minima, the phase retardation of the sample area corresponding to the pixel is obtained. The orientation of the principal axis of the sample area is simply the rotation angle of the polarizer where the intensity is the maximum. Due to lack of a standard reference specimen for birefringence, we checked the accuracy and precision of the microscope without a sample in the optical axis of the microscope. The accuracy was $0.005010 \mathrm{rad}$, and the precision was $0.000798 \mathrm{rad}$. The cytoskeletal structures of the breast cancer cells were clearly visible in the birefringence images obtained from the polarization microscope, while the same structures were not at all visible in the images obtained from a conventional microscope.

\section{Acknowledgments}

This research was supported by the Korean Ministry of Education, Science, and Technology (MEST) and the Korea Science and Engineering Foundation (KOSEF): I. Shin and D.
Kim under the Creative Research Initiatives Program (3D Nano Optical Imaging Systems Research Group, Contract Number R16-2006-068-01000-0) and S. Shin under the Bio Interphase Program (Bio Tool R\&D Group, Contract Number M10531020001-08N3102-00110). The help of Namhui Ahn with cell culture and preparation is appreciated.

\section{References}

1. H. B. Klein Brink, "Birefringence of the human crystalline lens in vivo," J. Opt. Soc. Am. A 8, 1788-1793 (1991).

2. D. G. Hunter, S. N. Patel, and D. L. Guyton, "Automated detection of foveal fixation by use of retinal birefringence scanning," Appl. Opt. 38, 1273-1279 (1999).

3. G. J. van Blokland, "Ellipsometry of the human retina in vivo: preservation of polarization," J. Opt. Soc. Am. A 2, 72-75 (1985).

4. D. J. Maitland and J. T. Walsh, "Interference-based linear birefringence measurement of thermally induced changes in collagen," Proc. SPIE 2134A, 304-308 (1994)

5. T. T. Tower and R. T. Tranquillo, "Alignment maps of tissues," Biophys. J. 81, 2954-2963 (2001).

6. L.-W. Jin, K. A. Claborn, M. Kurimoto, M. A. Geday, I. Maezawa, F. Sohraby, M. Estrada, W. Kaminksy, and B. Kahr, "Imaging linear birefringence and dichroism in cerebral amyloid pathologies," Proc. Natl. Acad. Sci. U.S.A. 100, 15294-15298 (2003).

7. R. Oldenbourg, E. D. Salmon, and P. T. Tran, "Birefringence of single and bundled microtubules," Biophys. J. 74, 645-654 (1998).

8. J. R. Kuhn, Z. Wu, and M. Poenie, "Modulated polarization microscopy: a promising new approach to visualizing cytoskeletal dynamics in living cells," Biophys. J. 80, 972-985 (2001).

9. K. Katoh, K. Hammer, P. J. Smith, and R. Oldenbourg, "Birefringence imaging directly reveals architectural dynamics of filamentous actin in living growth cones," J. Am. Soc. Cell Bio. 10, 197-210 (1999).

10. M. Shiribak and R. Oldenbourg, "Techniques for fast and sensitive measurements of two-dimensional birefringence distributions," Appl. Opt. 42, 3009-3017 (2003).

11. Acroptix S.A., personal correspondence with commercial LC retarder manufacturer.

12. Y. Park, K. Oh, U.-C. Paek, C. R. Kurkjian, and D. Y. Kim, "Residual stresses in a doubly clad fiber with depressed inner cladding(DIC)," J. Lightwave Technol. 17, 1823-1833 (1999).

13. Y. Park, "Stress measurement of an optical fiber," Doctor of Philosophy Dissertation Gwangju Institute of Science and Technology (2002).

14. J. A. DePasquale, "Rearrangement of the F-actin cytoskeleton in estradiol-treated MCF 7 breast carcinoma cells," Histochem. Cell Biol. 112, 341-350 (1999).

15. C. Gache, Y. Berthois, E. Cvitkovic, P.-M. Martin, and S. Saez, "Differential regulation of normal and tumoral breast epithelial cell growth by fibroblasts and 1,25-dihydroxyvitamin D," Breast Cancer Res. Treat. 55, 29-39 (1999). 